\title{
ON CERTAIN MODULI SPACES OF IDEAL SHEAVES AND DONALDSON-THOMAS INVARIANTS
}

\author{
Sheldon Katz ${ }^{1}$, Wei-PIng Li ${ }^{2}$, And Zhenbo Qin ${ }^{3}$
}

\begin{abstract}
We determine the structure of certain moduli spaces of ideal sheaves by generalizing an earlier result of the first author. As applications, we compute the (virtual) Hodge polynomials of these moduli space, and calculate the Donaldson-Thomas invariants of certain 3-folds with trivial canonical classes.
\end{abstract}

\section{Introduction}

Donaldson-Thomas theory was introduced in [DT, Tho] via integrals over the moduli spaces of semistable sheaves and via the theory of virtual fundamental cycles. It was further developed by Maulik-Pandharipande [MP] and Jun Li. In [LQ], we constructed rank-2 stable vector bundles over certain Calabi-Yau manifolds, and calculated the corresponding Donaldson-Thomas invariants. Recently, a rich interplay among Donaldson-Thomas theory, Gromov-Witten theory and Gopakumar-Vafa invariants has been proposed, and in certain cases, verified in [MNOP1, MNOP2, Kat, OP] (see the references there for other papers). For this interplay, Donaldson-Thomas theory is defined via the moduli spaces parametrizing ideal sheaves of 1-dimensional closed subschemes in a 3-fold, while Gromov-Witten theory is based on the moduli spaces of stable morphisms to the same 3 -fold.

In this note, we study the moduli spaces of ideal sheaves in certain 3 -fold $X$, and compute the corresponding Donaldson-Thomas invariants of $X$. Let $S$ be a smooth projective surface, and $\mu: X \rightarrow S$ be a Zariski-locally trivial fibration whose fibers are smooth irreducible curves of genus- $g$. Let $\beta \in H_{2}(X ; \mathbb{Z})$ be the class of a fiber. For two nonnegative integers $m$ and $n$, the moduli space

$$
\mathfrak{I}_{m(1-g)+n}(X, m \beta)
$$

parametrizes ideal sheaves $I_{Z} \subset \mathcal{O}_{X}$ where $Z$ denotes 1-dimensional closed subschemes of $X$ with $\chi\left(\mathcal{O}_{Z}\right)=m(1-g)+n$ and $[Z]=m \beta$. Here $[Z]$ is the class associated to the dimension-1 component (weighted by their intrinsic multiplicities) of $Z$. When $n=0$, the moduli space $\mathfrak{I}_{m(1-g)}(X, m \beta)$ is naturally identified with the Hilbert scheme $S^{[m]}$ which parametrizes the length- $m 0$-dimensional closed subschemes of $S$. Our main result is to determine $\mathfrak{I}_{m(1-g)+1}(X, m \beta)$.

\footnotetext{
Received by the editors June 15, 2005.

2000 Mathematics Subject Classification. Primary: 14C05; Secondary: 14D20, 14D21.

Key words and phrases. Moduli spaces, ideal sheaves, Donaldson-Thomas invariant.

${ }^{1}$ Partially supported by an NSF grant.

${ }^{2}$ Partially supported by the RGC CERG grant HKUST6114/02P and 601905.

${ }^{3}$ Partially supported by an NSF grant.
} 
Theorem 1.1. Identify $S^{[m]}$ and $\mathfrak{I}_{m(1-g)}(X, m \beta)$. Let $\widetilde{S^{[m]} \times X}$ be the blow-up of $S^{[m]} \times X$ along the universal curve. Then, we have an isomorphism

$$
\mathfrak{I}_{m(1-g)+1}(X, m \beta) \cong \widetilde{S^{[m]} \times X} .
$$

Moreover, the moduli space $\mathfrak{I}_{m(1-g)+1}(X, m \beta)$ is smooth.

The key step to prove the above theorem is to generalize a result of the first author to our Lemma 2.1 in Sect. 2 by assuming a canonical isomorphism between the blowup along the universal curve and the Grassmannian scheme associated to the ideal sheaf defining the universal curve. Indeed, the proof of Lemma 2.1 follows the same argument as in the proof of the Lemma 1 in [Kat]. The only difference is that instead of using local arguments, we apply the universal properties of various constructions. As an application to Theorem 1.1, we compute the (virtual) Hodge polynomials of the moduli space $\mathfrak{I}_{m(1-g)+1}(X, m \beta)$ using the results of Cheah and Ellingsrud-Strømme [Che, ES]. As another application, we show that when $X=S \times C$ with $K_{S}=0$ and $K_{C}=0$, the Donaldson-Thomas invariant of the 3-fold $X$ corresponding to the moduli space $\mathfrak{I}_{m(1-g)+1}(X, m \beta)$ is equal to zero.

Acknowledgments. The authors thank Jun Li for valuable discussions.

\section{The moduli space $\mathfrak{I}_{n}(X, \beta)$}

\subsection{The definition of $\mathfrak{I}_{n}(X, \beta)$.}

Let $X$ be a smooth projective complex variety of dimension $r$. For a fixed homology class $\beta \in H_{2}(X ; \mathbb{Z})$ and a fixed integer $n$, following [MNOP1, MNOP2], we define $\mathfrak{I}_{n}(X, \beta)$ to be the moduli space parametrizing all the 1-dimensional closed subschemes $Z$ of $X$ satisfying the conditions:

$$
\chi\left(\mathcal{O}_{Z}\right)=n, \quad[Z]=\beta
$$

where $[Z]$ is the class associated to the dimension- 1 component (weighted by their intrinsic multiplicities) of $Z$. It is also convenient to regard $\mathfrak{I}_{n}(X, \beta)$ as the moduli space parametrizing the corresponding ideal sheaves $I_{Z}$.

The degree- 0 moduli space $\mathfrak{I}_{n}(X, 0)$ is isomorphic to the Hilbert scheme $X^{[n]}$ parametrizing length- $n$ 0-dimensional closed subschemes of $X$. In general, when $\beta \neq 0$, the moduli space $\mathfrak{I}_{n}(X, \beta)$ is only part of the Hilbert scheme defined in terms of certain degree-1 Hilbert polynomial (see [Gro]). The Zariski tangent space of $\mathfrak{I}_{n}(X, \beta)$ at a point $[Z]$ is canonically isomorphic to

$$
\operatorname{Hom}\left(I_{Z}, \mathcal{O}_{Z}\right) \text {. }
$$

Let $K_{X}$ be the canonical class of $X$. By the Lemma 1 in [MNOP2], when $\operatorname{dim}(X)=3$, the virtual dimension of $\mathfrak{I}_{n}(X, \beta)$ is equal to

$$
-\left(\beta \cdot K_{X}\right) .
$$


2.2. A relation between $\mathfrak{I}_{1-g}(X, \beta)$ and $\mathfrak{I}_{2-g}(X, \beta)$.

Let $g \geq 0$. Assume that all the closed subschemes parametrized by $\mathfrak{I}_{1-g}(X, \beta)$ are curves of arithmetic genus $g$, and that the 1-dimensional components of all the closed subschemes parametrized by $\mathfrak{I}_{2-g}(X, \beta)$ are supported on the curves parametrized by $\mathfrak{I}_{1-g}(X, \beta)$. It follows that if $[Z] \in \mathfrak{I}_{2-g}(X, \beta)$, then there exists a unique $\left[Z^{\prime}\right] \in$ $\Im_{1-g}(X, \beta)$ together with an exact sequence:

$$
0 \rightarrow I_{Z} \rightarrow I_{Z^{\prime}} \rightarrow \mathcal{O}_{x} \rightarrow 0
$$

where $x$ is some point in $X$. Let $\mathcal{I}_{1-g}$ be the universal ideal sheaf over

$$
\mathfrak{I}_{1-g}(X, \beta) \times X,
$$

and let $\mathcal{C}_{1-g} \subset \mathfrak{I}_{1-g} \times X$ be the universal curve. Then, $\mathcal{I}_{1-g}=I_{\mathcal{C}_{1-g}}$.

Let $\mathbb{P}\left(\mathcal{I}_{1-g}\right)$ be the Grassmannian associated to the sheaf $\mathcal{I}_{1-g}$, and

$$
\tilde{\pi}: \mathbb{P}\left(\mathcal{I}_{1-g}\right) \rightarrow \mathfrak{I}_{1-g}(X, \beta) \times X
$$

be the natural projection. Then there exists a universal quotient

$$
\tilde{\pi}^{*} \mathcal{I}_{1-g} \rightarrow \mathcal{O}_{\mathbb{P}\left(\mathcal{I}_{1-g}\right)}(1) \rightarrow 0
$$

over $\mathbb{P}\left(\mathcal{I}_{1-g}\right)$. Let

$$
\pi: \mathfrak{I}_{1-g}\left(X, \widetilde{\beta) \times X} \rightarrow \mathfrak{I}_{1-g}(X, \beta) \times X\right.
$$

be the blow-up of $\mathfrak{I}_{1-g}(X, \beta) \times X$ along $\mathcal{C}_{1-g}$, and $E$ be the exceptional divisor. Then there exists a surjection over $\mathfrak{I}_{1-g}(X, \widetilde{\beta) \times X}$ :

$$
\pi^{*} \mathcal{I}_{1-g} \rightarrow \mathcal{O}_{\mathfrak{I}_{1-g}(X, \widetilde{\beta) \times X}}(-E) \rightarrow 0 .
$$

By the universal property of the Grassmannians, there exists a canonical morphism $\phi: \mathfrak{I}_{1-g}\left(X, \widetilde{\beta) \times X} \rightarrow \mathbb{P}\left(\mathcal{I}_{1-g}\right)\right.$ making a commutative diagram:

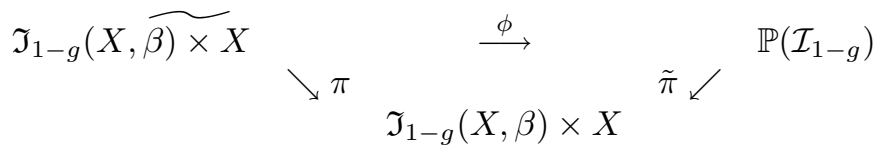

such that the pull-back of (2.6) via $\phi^{*}$ is (2.8). In particular,

$$
\phi^{*} \mathcal{O}_{\mathbb{P}\left(\mathcal{I}_{1-g}\right)}(1)=\mathcal{O}_{\widetilde{I}_{1-g}(X, \widetilde{\beta) \times X}}(-E) .
$$

Lemma 2.1. Assume that the closed subschemes parametrized by $\mathfrak{I}_{1-g}(X, \beta)$ are curves of arithmetic genus $g$, and that the 1-dimensional components of the closed subschemes parametrized by $\mathfrak{I}_{2-g}(X, \beta)$ are supported on the curves parametrized by $\mathfrak{I}_{1-g}(X, \beta)$. If the canonical morphism $\phi$ in (2.9) is an isomorphism, then

$$
\mathfrak{I}_{2-g}(X, \beta) \cong \mathfrak{I}_{1-g}(X, \widetilde{\beta) \times X} .
$$

Proof. For convenience, let $\mathfrak{I}_{n}=\mathfrak{I}_{n}(X, \beta)$. Let $\pi_{13}: \mathfrak{I}_{1-g} \times X \times X \rightarrow \mathfrak{I}_{1-g} \times X$ be the projection to the first and third factors. Let $\Delta_{X}$ be the diagonal in $X \times X$. Over $\mathfrak{I}_{1-g} \times X \times X$, we have the composition of the natural morphisms:

$$
\pi_{13}^{*} \mathcal{I}_{1-g} \rightarrow \mathcal{O}_{\mathfrak{I}_{1-g} \times X \times X} \rightarrow \mathcal{O}_{\mathfrak{I}_{1-g} \times \Delta_{X}} .
$$


Regarding (2.12) as a family of morphisms parametrized by $\mathfrak{I}_{1-g} \times X$, we see that (2.12) vanishes precisely along $\mathcal{C}_{1-g}$. So there is an induced morphism:

$$
\left(\pi \times \operatorname{Id}_{X}\right)^{*} \pi_{13}^{*} \mathcal{I}_{1-g} \rightarrow\left(\pi \times \operatorname{Id}_{X}\right)^{*} \mathcal{O}_{\mathfrak{I}_{1-g} \times \Delta_{X}}(-E)
$$

over $\widetilde{\mathfrak{I}_{1-g} \times} X \times X$. Note that $(2.13)$ is surjective since

$$
\begin{aligned}
& \left.\left(\pi \times \operatorname{Id}_{X}\right)^{*} \pi_{13}^{*} \mathcal{I}_{1-g}\right|_{\left(\pi \times \operatorname{Id}_{X}\right)^{-1}\left(\mathfrak{I}_{1-g} \times \Delta_{X}\right)} \\
= & \left.\left(\pi \times \operatorname{Id}_{X}\right)^{*} \pi_{12}^{*} \mathcal{I}_{1-g}\right|_{\left(\pi \times \operatorname{Id}_{X}\right)^{-1}\left(\mathfrak{I}_{1-g} \times \Delta_{X}\right)} \\
\rightarrow & \left.\mathcal{O}_{\mathfrak{I}_{1-g} \times X \times X}(-E)\right|_{\left(\pi \times \operatorname{Id}_{X}\right)^{-1}\left(\mathfrak{I}_{1-g} \times \Delta_{X}\right)},
\end{aligned}
$$

where the last map is surjective. Let $\tilde{\mathcal{I}}$ be the kernel of $(2.13)$. Then we have an exact sequence

$$
0 \rightarrow \tilde{\mathcal{I}} \rightarrow\left(\pi \times \operatorname{Id}_{X}\right)^{*} \pi_{13}^{*} \mathcal{I}_{1-g} \rightarrow\left(\pi \times \operatorname{Id}_{X}\right)^{*} \mathcal{O}_{\mathfrak{I}_{1-g} \times \Delta_{X}}(-E) \rightarrow 0
$$

over $\widetilde{\mathfrak{I}_{1-g} \times} X \times X$. Now $\tilde{\mathcal{I}}$ is flat over $\widetilde{\mathfrak{I}_{1-g} \times X}$ since the other two terms in (2.14) are. Also, the fibers of $\tilde{\mathcal{I}}$ over $\mathfrak{I}_{1-g} \times X$ are ideal sheaves parametrized by $\mathfrak{I}_{2-g}$.

Next, we check that $\tilde{\mathcal{I}} \subset \mathcal{O}_{\mathfrak{I}_{1-g} \times X \times X}$ is the universal ideal sheaf. Let $\mathcal{J} \subset \mathcal{O}_{T \times X}$ be a flat family of ideal sheaves in $\mathfrak{I}_{2-g}$ parametrized by $T$. Let $\mathcal{J}^{\prime}$ be the saturation of $\mathcal{J} \subset \mathcal{O}_{T \times X}$ (see Definition 1.1.5 in [HL]). Then, $\mathcal{J}^{\prime}$ is a flat family of ideal sheaves in $\mathfrak{I}_{1-g}$, and fits in an exact sequence

$$
0 \rightarrow \mathcal{J} \rightarrow \mathcal{J}^{\prime} \rightarrow\left(\operatorname{Id}_{T} \times f_{1}\right)_{*} \mathcal{L} \rightarrow 0
$$

for some morphism $f_{1}: T \rightarrow X$ and some line bundle $\mathcal{L}$ on $T$. The flat family $\mathcal{J}^{\prime}$ over $T \times X$ induces a morphism $f_{2}: T \rightarrow \mathfrak{I}_{1-g}$ such that

$$
\left(f_{2} \times \operatorname{Id}_{X}\right)^{*} \mathcal{I}_{1-g}=\mathcal{J}^{\prime} .
$$

By base-change, we have the commutative diagram:

$$
\begin{aligned}
& \begin{array}{ccc}
\mathbb{P}\left(\mathcal{J}^{\prime}\right) & \longrightarrow & \mathbb{P}\left(\mathcal{I}_{1-g}\right) \\
\downarrow & \downarrow
\end{array} \\
& T \stackrel{\mathrm{Id}_{T} \times f_{1}}{\longrightarrow} T \times X \stackrel{f_{2} \times \mathrm{Id}_{X}}{\longrightarrow} \quad \mathfrak{I}_{1-g} \times X .
\end{aligned}
$$

By $(2.15)$, we get a surjection $\left(\operatorname{Id}_{T} \times f_{1}\right)^{*} \mathcal{J}^{\prime} \rightarrow\left(\operatorname{Id}_{T} \times f_{1}\right)^{*}\left(\operatorname{Id}_{T} \times f_{1}\right)_{*} \mathcal{L} \rightarrow 0$. Since the natural morphism $\left(\operatorname{Id}_{T} \times f_{1}\right)^{*}\left(\operatorname{Id}_{T} \times f_{1}\right)_{*} \mathcal{L} \rightarrow \mathcal{L}$ is surjective, we obtain

$$
\left(\operatorname{Id}_{T} \times f_{1}\right)^{*} \mathcal{J}^{\prime} \rightarrow \mathcal{L} \rightarrow 0
$$

over $T$. By the universal property of $\mathbb{P}\left(\mathcal{J}^{\prime}\right)$, we obtain a commutative diagram

$$
\begin{array}{cccc} 
& \mathbb{P}\left(\mathcal{J}^{\prime}\right) & \longrightarrow & \mathbb{P}\left(\mathcal{I}_{1-g}\right) \\
& \downarrow & & \downarrow \\
\stackrel{\operatorname{Id}_{T} \times f_{1}}{\longrightarrow} & T \times X & \stackrel{f_{2} \times \operatorname{Id}_{X}}{\longrightarrow} & \mathfrak{I}_{1-g} \times X .
\end{array}
$$

Thus the morphism $f_{2} \times f_{1}: T \rightarrow \mathfrak{I}_{1-g} \times X$ can be lifted to a morphism

$$
f: T \longrightarrow \mathbb{P}\left(\mathcal{I}_{1-g}\right) \stackrel{\phi^{-1}}{\longrightarrow} \widetilde{\mathfrak{I}_{1-g}} \times X .
$$


Finally, we apply the pull-back $\left(f \times \operatorname{Id}_{X}\right)^{*}$ to $(2.14)$. Using (2.10) and the property of the morphism $f$, we obtain a commutative diagram:

$$
\begin{aligned}
& 0 \rightarrow\left(f \times \operatorname{Id}_{X}\right)^{*} \tilde{\mathcal{I}} \rightarrow \mathcal{J}^{\prime} \rightarrow\left(f \times \operatorname{Id}_{X}\right)^{*}\left(\pi \times \operatorname{Id}_{X}\right)^{*} \mathcal{O}_{\mathfrak{I}_{1-g} \times \Delta_{X}}(-E) \rightarrow 0 \\
& \| \\
& 0 \rightarrow \quad \mathcal{J} \quad \rightarrow \mathcal{J}^{\prime} \rightarrow \quad \rightarrow \quad\left(\operatorname{Id}_{T} \times f_{1}\right)_{*} \mathcal{L} \quad \rightarrow 0
\end{aligned}
$$

over $T \times X$. In particular, there exists an injection

$$
0 \rightarrow\left(f \times \operatorname{Id}_{X}\right)^{*} \tilde{\mathcal{I}} \stackrel{\psi}{\rightarrow} \mathcal{J} .
$$

Since both $\left(f \times \operatorname{Id}_{X}\right)^{*} \tilde{\mathcal{I}}$ and $\mathcal{J}$ are flat families of ideal sheaves in $\mathfrak{I}_{2-g}(X, \beta)$, we conclude that the morphism $\psi$ is an isomorphism.

Remark 2.2. If the universal curve $\mathcal{C}_{1-g} \subset \mathfrak{I}_{1-g} \times X$ is a local complete intersection, then $\left(\mathcal{I}_{1-g}\right)^{n} \cong \operatorname{Sym}^{n}\left(\mathcal{I}_{1-g}\right)$, and so the canonical morphism $\phi$ in (2.9) is an isomorphism. Hence Lemma 2.1 indeed generalizes the Lemma 1 in [Kat].

\subsection{Some comments on $\mathfrak{I}_{3-g}(X, \beta)$.}

In this subsection, we make a few comments about the moduli space $\mathfrak{I}_{3-g}(X, \beta)$. As in the previous subsection, we assume that all the closed subschemes parametrized by $\mathfrak{I}_{1-g}(X, \beta)$ are curves of arithmetic genus $g$, and that the 1-dimensional components of all the closed subschemes parametrized by $\mathfrak{I}_{3-g}(X, \beta)$ are supported on the curves parametrized by $\mathfrak{I}_{1-g}(X, \beta)$. Therefore, if $[Z] \in \mathfrak{I}_{3-g}(X, \beta)$, then there exists a unique $\left[Z^{\prime}\right] \in \mathfrak{I}_{1-g}(X, \beta)$ together with an exact sequence:

$$
0 \rightarrow I_{Z} \rightarrow I_{Z^{\prime}} \rightarrow Q \rightarrow 0
$$

where $Q$ is a torsion sheaf on $X$ with $\ell(Q)=2$.

Lemma 2.3. Let $Q$ be a torsion sheaf on $X$ from (2.20). Then, either $Q \cong \mathcal{O}_{\xi}$ for some $\xi \in X^{[2]}$, or $Q \cong \mathcal{O}_{x} \oplus \mathcal{O}_{x}$ for some point $x \in Z^{\prime}$.

Proof. Our lemma follows immediately from the following claim.

Claim. Let $Q$ be a length-2 torsion sheaf supported on at most two points of $X$. Then, either $Q \cong \mathcal{O}_{\xi}$ for some $\xi \in X^{[2]}$, or $Q \cong \mathcal{O}_{x} \oplus \mathcal{O}_{x}$ for some $x \in X$.

Proof. If $\operatorname{Supp}(Q)=\left\{x_{1}, x_{2}\right\}$ with $x_{1} \neq x_{2}$, then $Q \cong \mathcal{O}_{x_{1}} \oplus \mathcal{O}_{x_{2}}$ and we are done. In the following, we assume that $\operatorname{Supp}(Q)=\{x\}$ for some $x \in X$. We further assume that $X=\operatorname{Spec}(A)$ is affine and $Q$ is an $A$-module by abuse of notations.

Take a nonzero $v \in Q$ and define an $A$-module homomorphism $\varphi: A \rightarrow Q$ by sending 1 to $v$. Then there exists an ideal $J \subset A$ such that the induced homomorphism $\bar{\varphi}: A / J \rightarrow Q$ is injective. If $\bar{\varphi}$ is also surjective, then $Q \cong \mathcal{O}_{\xi}$ for $\xi \in X^{[2]}$. If $\bar{\varphi}$ is not surjective, then we have an exact sequence

$$
0 \rightarrow A / J \rightarrow Q \rightarrow Q^{\prime} \rightarrow 0
$$

where $Q^{\prime}$ and $A / J$ must be of length one. Therefore $A / J \cong \mathcal{O}_{x}$ and $Q^{\prime} \cong \mathcal{O}_{x}$. Hence the minimal number of generators of $Q$ is at most two.

If $Q$ is generated by a single element, say $v_{0}$, then by replacing the above $v \in Q$ by $v_{0} \in Q$, we conclude that $Q \cong \mathcal{O}_{\xi_{0}}$ for $\xi_{0} \in X^{[2]}$. 
Now we are left with the case when the minimal number of generators for $Q$ is two. Assume that $v_{1}$ and $v_{2}$ generate $Q$. We define two homomorphisms:

$$
\begin{array}{ll}
\varphi_{1}: A \rightarrow \mathcal{Q}, & \varphi_{1}(1)=v_{1} ; \\
\varphi_{2}: A \rightarrow \mathcal{Q}, \quad \varphi_{2}(1)=v_{2} .
\end{array}
$$

Then $\varphi_{1}$ and $\varphi_{2}$ induce injective homomorphisms:

$$
\bar{\varphi}_{1}: A / J_{1} \rightarrow Q \quad \text { and } \quad \bar{\varphi}_{2}: A / J_{2} \rightarrow Q
$$

respectively. Note that both $A / J_{1}$ and $A / J_{2}$ must be of length-1. So $A / J_{1} \cong \mathcal{O}_{x}$ and $A / J_{2} \cong \mathcal{O}_{x}$. Define $\psi: A / J_{1} \oplus A / J_{2} \rightarrow Q$ by putting

$$
\psi(a, b)=a v_{1}+b v_{2} .
$$

Then $\psi$ is surjective. Since both $Q$ and $A / J_{1} \oplus A / J_{2}$ have length two, $\psi$ must be an isomorphism. Therefore, we see that $Q \cong \mathcal{O}_{x} \oplus \mathcal{O}_{x}$.

Conversely, we can show that if $\left[Z^{\prime}\right] \in \mathfrak{I}_{1-g}(X, \beta)$ and $Z^{\prime}$ is smooth, then both types of $Q$ in Lemma 2.3 can arise from (2.20). Indeed, fix a point $x \in Z^{\prime}$. Then there is exactly one surjection $I_{Z^{\prime}} \rightarrow \mathcal{O}_{x} \oplus \mathcal{O}_{x} \rightarrow 0$ up to isomorphism classes of $\mathcal{O}_{x} \oplus \mathcal{O}_{x}$. Next, let $\xi \in X^{[2]}$ with $\operatorname{Supp}(\xi)=\{x\}$. If $\xi$ points to the tangent direction of $Z^{\prime}$ at $x$, then the set of surjections $I_{Z^{\prime}} \rightarrow \mathcal{O}_{\xi} \rightarrow 0$ up to isomorphisms has dimension 2. If $\xi$ is transverse to $Z^{\prime}$ at $x$, then the set of surjections $I_{Z^{\prime}} \rightarrow \mathcal{O}_{\xi} \rightarrow 0$ up to isomorphisms has dimension 1. However, it is not clear how to globalize these local data into a complete description of $\mathfrak{I}_{3-g}(X, \beta)$ in terms of $\mathfrak{I}_{1-g}(X, \beta)$.

\section{Applications}

In this section, we adopt the following basic assumptions.

Assumption 3.1. We assume that $X$ admits a Zariski-locally trivial fibration

$$
\mu: X \rightarrow S
$$

where $S$ is a smooth surface, the fibers are smooth irreducible curves of genus- $g$, and $\beta \in H_{2}(X ; \mathbb{Z})$ is the class of a fiber. For $m, n \geq 0$, we let

$$
\mathfrak{I}_{m, n}:=\mathfrak{I}_{m(1-g)+n}(X, m \beta) .
$$

Note that the elements in $\mathfrak{I}_{m, 0}$ correspond to the ideal sheaves of the form $\mu^{*} I_{\xi}$ for some $\xi \in S^{[m]}$. Hence there exists a bijective morphism $S^{[m]} \rightarrow \mathfrak{I}_{m, 0}$. It is well-known that the Hilbert scheme $S^{[m]}$ is smooth. Combining with $(2.2)$, one checks that the moduli space $\mathfrak{I}_{m, 0}$ is smooth and that

$$
\mathfrak{I}_{m, 0} \cong S^{[m]}
$$

Theorem 3.2. Identify $S^{[m]}$ and $\mathfrak{I}_{m, 0}$ by (3.3). Let $\widetilde{S^{[m]} \times X}$ be the blow-up of $S^{[m]} \times X$ along the universal curve. Then, we have an isomorphism

$$
\mathfrak{I}_{m, 1} \cong \widehat{S^{[m]} \times X} .
$$

Moreover, the moduli space $\mathfrak{I}_{m, 1}=\mathfrak{I}_{m(1-g)+1}(X, m \beta)$ is smooth. 
Proof. We will apply Lemma 2.1 to the present situation. First of all, note that the 1-dimensional components of the closed subschemes parametrized by $\mathfrak{I}_{m, 1}$ are supported on the curves parametrized by $\mathfrak{I}_{m, 0}$. Next, let $\mathcal{Z}_{m} \subset S^{[m]} \times S$ be the universal codimension-2 subscheme. Set-theoretically,

$$
\mathcal{Z}_{m}=\left\{(\xi, x) \in S^{[m]} \times S \mid x \in \operatorname{Supp}(\xi)\right\} .
$$

Let $\widetilde{S^{[m]} \times S}$ be the blow-up of $S^{[m]} \times S$ along $\mathcal{Z}_{m}$. By the results in [ES], $\widetilde{S^{[m]} \times S}$ is smooth and there exists a canonical isomorphism:

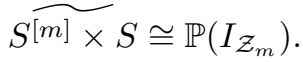

Now the universal curve $\mathcal{C}_{m} \subset S^{[m]} \times X$ is $\left(\operatorname{Id}_{S[m]} \times \mu\right)^{*} \mathcal{Z}_{m}$. Since $\mu$ is Zariski-locally trivial, we obtain canonical isomorphisms:

$$
\begin{aligned}
\widetilde{S^{[m]} \times X} & \cong\left(\widehat{S^{[m]} \times S}\right) \times{ }_{S^{[m]} \times S}\left(S^{[m]} \times X\right) \\
& \cong \mathbb{P}\left(I_{\mathcal{Z}_{m}}\right) \times{ }_{S^{[m]} \times S}\left(S^{[m]} \times X\right) \\
& \cong \mathbb{P}\left(I_{\mathcal{C}_{m}}\right) .
\end{aligned}
$$

By Lemma 2.1, we have an isomorphism $\mathfrak{I}_{m, 1} \cong \widetilde{S^{[m]} \times X}$. Note from the isomorphism (3.6) that $\widetilde{S^{[m]} \times X}$ is smooth. Hence $\mathfrak{I}_{m, 1}$ is smooth as well.

Corollary 3.3. Let $e(\cdot ; s, t)$ denote the (virtual) Hodge polynomial. Then,

$$
\sum_{m=0}^{+\infty} e\left(\Im_{m, 1} ; s, t\right) q^{m}=\frac{q}{1-s t q} \cdot e(X ; s, t) \cdot \prod_{k=1}^{+\infty} \prod_{i, j}\left(\frac{1}{1-s^{i+k-1} t^{j+k-1} q^{k}}\right)^{e^{i, j}(S)}
$$

where $e^{i, j}(S)=(-1)^{i+j} h^{i, j}(S)$ and $h^{i, j}(S)$ denotes the Hodge numbers of $S$.

Proof. Since $\mu$ is Zariski-locally trivial, we see from (3.6) that the natural projection

$$
\widehat{S^{[m]} \times X} \rightarrow \widetilde{S^{[m]} \times S}
$$

is a Zariski-locally trivial fibration with fibers being isomorphic to the fibers $C$ of $\mu$. Thus, $e\left(\widehat{S^{[m]} \times X} ; s, t\right)=e(C ; s, t) \cdot e\left(\widehat{S^{[m]} \times S} S s, t\right)$. By Theorem 3.2,

$$
\sum_{m=0}^{+\infty} e\left(\mathfrak{I}_{m, 1} ; s, t\right) q^{m}=e(C ; s, t) \cdot \sum_{m=0}^{+\infty} e\left(\widetilde{S^{[m]} \times S} ; s, t\right) q^{m} .
$$

By the Proposition 2.2 in $[\mathrm{ES}], \widehat{S^{[m]} \times S}$ is isomorphic to the incidence variety:

$$
S_{m, m+1}=\left\{(\eta, \xi) \in S^{[m]} \times S^{[m+1]} \mid \eta \subset \xi\right\} .
$$

The (virtual) Hodge polynomial of $S_{m, m+1}$ has been computed by Cheah:

$$
\sum_{m=0}^{+\infty} e\left(S_{m, m+1} ; s, t\right) q^{m}=\frac{q}{1-s t q} \cdot e(S ; s, t) \cdot \prod_{k=1}^{+\infty} \prod_{i, j}\left(\frac{1}{1-s^{i+k-1} t^{j+k-1} q^{k}}\right)^{e^{i, j}(S)}
$$

(see p.485 in [Che]). Combining this with (3.7) completes the proof. 
Remark 3.4. It is well-known that $e(\cdot ; 1,1)$ is equal to the topological Euler number $\chi(\cdot)$. Therefore, we see from Corollary 3.3 that

$$
\sum_{m=0}^{+\infty} \chi\left(\mathfrak{I}_{m, 1}\right) q^{m}=\frac{q}{1-q} \cdot \chi(X) \cdot \prod_{k=1}^{+\infty}\left(\frac{1}{1-q^{k}}\right)^{\chi(S)} .
$$

It is also interesting to note from (3.3) and Göttsche's formula in [Got] that

$$
\sum_{m=0}^{+\infty} \chi\left(\Im_{m, 0}\right) q^{m}=\sum_{m=0}^{+\infty} \chi\left(S^{[m]}\right) q^{m}=\prod_{k=1}^{+\infty}\left(\frac{1}{1-q^{k}}\right)^{\chi(S)} .
$$

Let $X=S \times C$ where $C$ is an elliptic curve and $S$ is a smooth surface with $K_{S}=0$. Let $\mu: X \rightarrow S$ be the first projection. Then, $K_{X}=0$. By (2.3), the virtual dimension of the moduli space $\mathfrak{I}_{m, n}$ is zero. The corresponding Donaldson-Thomas invariant is an integer. We denote this Donaldson-Thomas invariant by

$$
N_{m, n} .
$$

Corollary 3.5. Let $X=S \times C$ where $C$ is an elliptic curve and $S$ is a smooth surface with $K_{S}=0$. Then, $N_{m, 1}=0$ for every $m \geq 0$.

Proof. Since $K_{X}=0$ and the moduli space $\mathfrak{I}_{m, 1}$ is smooth, the obstruction bundle over $\mathfrak{I}_{m, 1}$ is the dual of the tangent bundle of $\mathfrak{I}_{m, 1}$ (see [Tho]). Hence

$$
N_{m, 1}=(-1)^{\operatorname{dim} \mathfrak{I}_{m, 1}} \cdot \chi\left(\mathfrak{I}_{m, 1}\right) .
$$

Since $\chi(X)=0$, we see from (3.9) that $\chi\left(\Im_{m, 1}\right)=0$. Therefore, $N_{m, 1}=0$.

Conjecture 3.6. Let $X=S \times C$ where $C$ is an elliptic curve and $S$ is a smooth surface with $K_{S}=0$. Then, $N_{m, n}=0$ for all $m \geq 0$ and $n \geq 1$.

\section{References}

[Che] J. Cheah, The virtual Hodge polynomials of nested Hilbert schemes and related varieties, Math. Z. 227 (1998), no .3, 479-504.

[DT] S.K. Donaldson, R.P. Thomas, Gauge theory in higher dimensions, In: The geometric universe (Oxford, 1996), 31-47. Oxford Univ. Press, Oxford, 1998.

[ES] G. Ellingsrud, S.A. Strømme, An intersection number for the punctual Hilbert scheme of a surface, Trans. Amer. Math. Soc. 350 (1998), no. 6, 2547-2552.

[Got] L. Göttsche, The Betti numbers of the Hilbert scheme of points on a smooth projective surface, Math. Ann. 286 (1990), no. 1-3, 193-207.

[Gro] A. Grothendieck, Techniques de construction et théorèmes d'existence en géométrie algébrique IV, Sem. Bourbaki 221, 13. 1960-1961.

[HL] D. Huybrechts, M. Lehn, The geometry of moduli spaces of sheaves, Aspects of Mathematics, E31. Friedr. Vieweg \& Sohn, Braunschweig, 1997.

[Kat] S. Katz, Gromov-Witten, Gopakumar-Vafa, and Donaldson-Thomas invariants of Calabi-Yau threefolds, Snowbird lectures on string geometry, 43-52, Contemp. Math., 401, Amer. Math. Soc., Providence, RI, 2006.

[LQ] W.-P. Li, Z. Qin, Stable rank-2 bundles on Calabi-Yau manifolds, Internat. J. Math. 14 (2003), no. 10, 1097-1120.

[MNOP1] D. Maulik, N. Nekrasov, A. Okounkov, R. Pandharipande, Gromov-Witten theory and Donaldson-Thomas theory, math.AG/0312059.

[MNOP2] D. Maulik, N. Nekrasov, A. Okounkov, R. Pandharipande, Gromov-Witten theory and Donaldson-Thomas theory, II, math.AG/0406092.

[MP] D. Maulik, R. Pandharipande, Foundation of Donaldson-Thomas theory, In preparation. 
[OP] A. Okounkov, R. Pandharipande, Quantum cohomology of the Hilbert schemes of points in the plane, math.AG/0411210.

[Tho] R.P. Thomas, A holomorphic Casson invariant for Calabi-Yau 3-folds, and bundles on K3 fibrations, J. Differential Geom. 54 (2000), no. 2, 367-438.

Department of Mathematics, University of Illinois, Urbana, IL 61801

E-mail address: katz@math.uiuc.edu

Department of Mathematics, HKust, Clear Water Bay, Kowloon, Hong Kong

E-mail address: mawpli@ust.hk

Department of Mathematics, University of Missouri, Columbia, MO 65211, USA

E-mail address: zq@math.missouri.edu 